\title{
A STORAGE PROCESS OF THE MAGNETIC ENERGY IN THE SPACE ACTIVE REGIONS AND STELLAR ATMOSPHERE
}

\author{
LI ZHONGYUAN \\ Dept. of Earth and Space Science \\ University of Science and Technology of China \\ Anhui, Hefei 230029 \\ People's Republic of China
}

A few authors (Barnes and Sturrock, 1972; Ma, 1977; Svestka, 1977) have calculated the quantitative relationship between the static force-free field connecting the magnetic field and the twisting processes. They pointed out that the potential magnetic field without the current may be twisted into the force-free field with the enhanced current produced by the plasma rotation. $\mathrm{Li}$ et al. (1982) and $\mathrm{Li}$ and $\mathrm{Hu}$ (1984) have stated that the processes should be unsteady, and especially that they should not be static. The magnetic Reynold number is usually much larger than 100 in stellar atmosphere ( $\mathrm{Li}$ et al., 1982). We adopt the following MHD equations:

$$
\begin{aligned}
& \partial \underline{B} / \partial t=\nabla \times(\underline{V} \times \underline{B})+\eta \nabla^{2} \underline{B}, \\
& \nabla \cdot \underline{B}=0, \\
& \nabla \times \underline{B}=\alpha(t, \underline{r}) \underline{B} .
\end{aligned}
$$

where the force-free factor $\alpha(t, \underline{r})$ depends on both, $t$ and $\underline{r}$. According to the kinematical momentum conservation, the following constraint is easily obtained:

$$
\underline{B} \cdot[\partial \underline{V} / \partial t+(\underline{V} \cdot \nabla) \underline{V}+\nabla p / \rho]=0 \text {, }
$$

where $\mathrm{V}=(\mathrm{u}, \mathrm{v}, \mathrm{w})$ is the velocity field in the cylindrical coordinates. When studying the evolution of the kinematical force-free field, the influence of a reasonable flow on the variations of the magnetic field should be taken into account. After some reasonable simplification we deduce the specific expression of the variation law of the toroidal magnetic energy

$$
W_{\theta}=\underline{B}_{\theta}^{2} / 8 \pi=\left(1 / r^{2}\right)\left[f^{2}(t)+C_{0}^{2} r^{2} \exp \left(-2 \lambda_{0} z\right) J_{1}^{2}\left(\beta_{0} r\right)\right],
$$

where $J_{1}$ is the Bessel function of the first order. In the active region, magnetic energy including the term of a twisted effect $f(t)$ is larger than that of the static force-free field.

The regular region of the solutions should, therefore, exclude the 
region near the origin $\mathbf{r}=0$. If the application region includes the region near the origin $r=0$, the regular condition requires $f(t)=0$. In this case both the poloidal and toroidal magnetic fields will be unable to change with time $t$. The continuous configuration of the force-free field in the ideal plasma can, therefore, not be maintained by the pure unsteady plasma rotation. The discussion about the energy storage by a pure azimuthal motion in the active regions of solar and stellar atmosphere is thus not a perfect approach. We should, furthermore, consider the unsteady poloidal flow field and the unsteady coupling processes between the magnetic field and both the toroidal and the poloidal velocity components.

Besides the rotation plasma motion, therefore, the poloidal plasma motion should play an important role. An assorted poloidal plasma motion exists in the active regions. Yeh (1982), Hu (1983), Li (1986) and $\mathrm{Li}$ et al. (1988) discussed the features on some poloidal plasma motion. From equations we know that the poloidal velocity field cannot be zero and is also unsteady in general. Due to the unsteady poloidal plasma motion, now we get the following force-free equation and boundary conditions:

$$
\begin{array}{ll}
\left(\partial^{2} / \partial r^{2}-1 / r \cdot \partial / \partial r+\partial^{2} / \partial z^{2}\right) A+\alpha^{2}(t) A=0, & A(r, L, t)=f_{2}(t, r), \\
A(0,0, t)=f_{1}(t, r), & A(0, t)=0, \quad A(0, z, t)=0,
\end{array}
$$

where $\alpha$ and the magnetic potential A are functions of time $t$. Through deduction the solution of eq. $(6)-(8)$ can be obtained, and the magnetic potential $A(r, z, t)$ has a more complex form. Analyzing $A(r, z, t)$ we know that the toroidal magnetic energy exists when the force-free factor $\alpha$ increases with time $t$. Due to the addition of the poloidal flow, an unsteady process which causes the change of $\alpha$ with time can be maintained in the active regions. The change of $\alpha$ with time $t$ mirrors some effects of the plasma motion in the solar and stellar atmosphere. Due to the poloidal plasma motion, the kinetic energy and the internal energy of the plasma uner the lower levels may be transferred to the upper levels of the active regions. The coupling process between the magnetic field and the flow field will cause the enhancement of the force-free factor $\alpha$ with time $t$. The enhancing factor $\alpha$ will impel the increase in the toroidal magnetic field. If also plasma rotation motion exists at the same time, the toroidal magnetic field can increase even more. In this case the energy may be stored up in the active regions. It is possible that the active regions become unstable on the suitable condition.

In fact, even if there are no twisted magnetic lines of force in the active regions, the energy of the force-free magnetic field can still be increased and stored. For example, when the plasma flow field are $u=0$, $\mathrm{v}=0, \mathrm{w}=\mathrm{w}(\mathrm{t})=0$, we may also obtain the contribution of the magnetic field $B_{r}, B_{\theta}, B_{z}$ and also get the magnetic energy of the active region under these circumstances, as

$$
W=M \exp [2 b \cdot s(t)][1-\exp (-2 b L)]\left[J_{1}^{2}(a R)+J_{0}^{2}(a R)\right],
$$

where $M=N_{0} a^{2} R^{2} / 16 b$ are a suitable constant which can be determined. 
The active region limits are taken as $r \leq R, 0 \leq z \leq L$. Eq. (9) shows that the magnetic energy changes with time t. From Eq. (9) we can know that the magnetic energy increases when the plasma goes up, the energy of the lower levels is transferred to the upper levels by the plasma motion. Due to the features of the poloidal motion in the solar and stellar atmosphere, it is possible that the "up motion" dominates on the suitable condition. Then a sizeable fraction of the energy may be stored in the active region under these circumstances.

When the force-free factor $\alpha$ increases, the magnetic lines of force are twisted. It might be a general storage process of the energy in the space active regions and the stellar atmosphere. However, as long as the energy under the lower levels can be transferred to the upper levels, the energy can be stored as well, even if there is no twisted line of force. of course, the storage of the energy of the force-free field should all be caused by an outside energy flow.

\section{References}

Barnes, C.W. and Sturrock, P.A. (1972) Astrophys. J. 174, 659.

Hu, W.R. (1983) Solar Phys. 83, 195.

Li, Z. et al. (1982) J. USTC Suppl. 1, 137.

Li, Z. and Hu, W.R. (1984) Mon. J. Sci. 29, 980.

Li, Z. (1986) ICTP/85/No. 158.

Li, Z. et al. (1988) Astrophys. Space Sci. 146, 59.

Ma, Y. (1977) The Proceeding of Huangshan Conference on the Astrophysics, Sci. Press, Beijing, China, p. 202.

Svestka, Z. (1977) in Solar Flares, Reidel Publ. Co., Dordrecht, p. 300.

Yeh, T. (1982) Solar Phys. 78, 287. 